\title{
The Effects of Segmentation Techniques in Digital Image Based Identification of Ethiopian Paper Currency
}

\author{
Solomon Wondaya Guangul \\ Department of Statistics, College of Science, Bahir Dar University
}

\begin{tabular}{|c|c|}
\hline Article Info & ABSTRACT \\
\hline Article history: & Paper and coin are the two most common currencies in all over the world. In \\
\hline Received Dec 13, 2017 & $\begin{array}{l}\text { Ethiopla also paper and coin currency are used for medium of exchange. } \\
\text { This paper presents the comparative study of segmentation techniques }\end{array}$ \\
\hline Revised Mar 3, 2018 & towards Ethiopian paper currency classification. Otsu, FCM and K-means \\
\hline Accepted Aug 21, 2018 & $\begin{array}{l}\text { segmentation techniques are considered for this study and BPNN is used for } \\
\text { classification of currencies. For the classification, images are collected from }\end{array}$ \\
\hline Keywords: & $\begin{array}{l}\text { commercial bank of Ethiopia and Dashen Bank; for our data set, a total of } \\
500 \text { images samples were collected. From these images, } 91.2 \% \text { accuracy is }\end{array}$ \\
\hline BPNN & $\begin{array}{l}\text { achieved when Otsu segmentation is used on BPNN with TANH learning } \\
\text { function. }\end{array}$ \\
\hline
\end{tabular}

Copyright () 2018 Institute of Advanced Engineering and Science. All rights reserved.

\section{Corresponding Author:}

Solomon Wondaya Guangul,

Department of Statistics,

College of Science, Bahir Dar University,

Email: solomonw@bdu.edu.et

\section{INTRODUCTION}

Currency is a medium of exchange that is used in every activity of human life. Paper and coin are the two most common currencies in all over the world. In Ethiopia also paper and coin currency is used for medium of exchange, as shown in Figure 1. Currently Ethiopian currency includes coins such as one cent, five cent, ten cent, twenty-five cent, fifty cent and one Birr cent and paper currency one Birr, five Birr, ten Birr, fifty Birr and hundred Birr [1]. Due to highly sophisticated devices, currency notes counterfeiting is the major problem around the world and it is very difficult to identify the forged notes from the actual notes. With the help of computer vision, it is better to identify forged notes from actual currency notes as compared to human eyes [2].

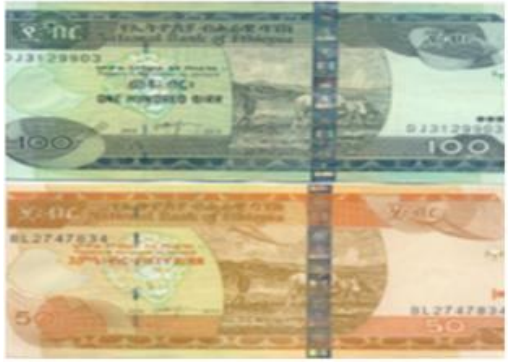

(a)

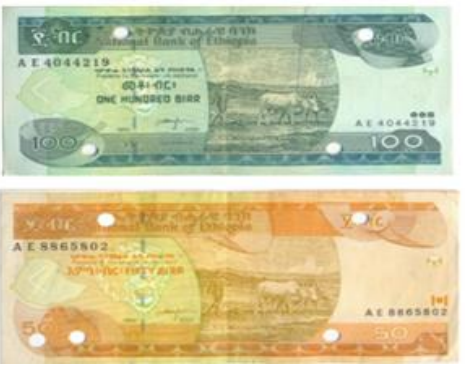

(b)

Figure 1. Actual Ethiopian paper currency (a) \& Fake Ethiopian paper currency (b) 
Currently, computer vision is gradually finding applications in different problem domains [3]. A technology of Computer vision applications requires different image pre-processing techniques among these; segmentation technique is the backbone in image processing field. Segmentation technique is used to extract the representative regions of the image. To identify these features the given image has to be partitioned that are visually different [4]. There is no particular image segmentation technique that is appropriate to all image processing areas [5]. Therefore, this research paper focused on the comparative study of segmentation techniques towards Ethiopian currency recognition system. The following image segmentation techniques are presented in this paper:

\subsection{Otsu Image Segmentation}

Otsu Image Segmentation the most commonly used technique to partition the image as foreground and background. Otsu's method involves iterating through all the possible threshold values and calculating a measure of spread for the pixel levels each side of the threshold, i.e. the pixels that either falls in foreground or background. The aim is to find the threshold value where the sum of foreground and background spreads is at its minimum [6].

Algorithm steps:

1) Compute histogram and probabilities of each intensity level.

2) Set up initial class probability and initial class means.

3) Step through all possible thresholds maximum intensity.

4) Update qi and $\mu \mathrm{i}$.

5) Compute between class variance.

6) Desired threshold corresponds to the maximum value of between class variance.

\subsection{FCM image segmentation}

In FCM, it is possible for a data sample to belong to multiple clusters at the same time. The similarity is indicated by the membership value. In FCM a data sample is assigned with a membership value based on its similarity with the cluster center. The membership values are between 0 to 1 and more the similarity, higher the membership value. Defuzzification is applied at the end of the clustering process to decide the clustering. FCM is a repetitive algorithm and the solution is achieved by repetitively updating the cluster center and membership value [7].

\subsection{K-means image segmentation}

$\mathrm{K}-\mathrm{Means}$ is least-square partitioning methods that divide a collection of objects into $\mathrm{K}$ groups. The algorithm iterates over two steps:

1) Compute the mean of each cluster.

2) Compute the distance of each point from each cluster by computing its distance from the corresponding cluster mean. Assign each point to the cluster it is nearest to.

Iterate over the above two steps till the sum of squared within group errors cannot be lowered any more. The initial assignment of points to clusters can be done randomly. In the iterations, the algorithm tries to minimize the sum, over all groups, of the squared within group errors, which are the distances of the points to the respective group means. Convergence is reached when the objective function (i.e., the residual sum-ofsquares) cannot be lowered any more [8].

Yao Yu \& Xuesong Suo conducted a study to detect smoothness of bottle cap. In this paper the author have used MATLAB and C language as a tool for image segmentation, enhancement, filtering and other processing of smoothness of cap [9].

On research [10], the authors proposed lettuce image segmentation. In this paper the authors described the traditional 2-D maximum entropy algorithm has some faults, such as low accuracy of segmentation, slow speed, and poor anti-noise ability. All the studies showed that Image segmentation is the basic parts in computer vision processing. So this research paper focused on the performance analysis of segmentation techniques towards to classification of Ethiopian paper currency.

\section{RESEARCH METHOD}

In this study, scanner HP scan jet pro 4500 and camera canon EOS 600d are used to capture the image. To minimize noises like lighting and blurred image effects while capturing the images scanners are effective. Therefore, scanner is used in this study. In order to have a good dataset form all perspective new, old and aperture paper currencies for both actual and fake paper currencies are considered. A total of 5 paper currency notes type each having 100 are considered for this study. Once the images are collected, preprocessing steps are performed to achieve the goal of the study through MATLAB, 2014. 


\section{IMAGE PROCESSING SYSTEM}

Paper currencies are scanned with a suitable resolution and are stored as a binary image. Before being analyzed image undergoes some pre-processing, including rehabilitation, smoothing and normalization. The pre-processing is carried out in order to improve the quality of image to be processed [11]. Image processing and pattern recognition performed by analyzing the image of Ethiopian paper currency [12]. The phase of activities for identification of paper currency consists of the following components:

The image is acquired from the scanner which is used as input image. The image is usually stored in jpg, tiff or png file format. In this research paper 80 X80, 360 X 360 and 512 X512 images are tested. Even though, pre-processing is slow, when the size of image increases, segmentation become effective. For that reason, the images are resized to 512 X512 [13].

Pre-processing are those operations that are normally required prior to the main data analysis and extraction of information. The aim of image pre-processing is to suppress undesired distortions or enhance some image features that are important for further processing or analysis [14].

Image segmentation is an important component of image processing technique that determines the accuracy of the system. Image segmentation is defined as the partitioning of an image into none overlapping, constituent regions that are homogenous with respect to some characteristic such as intensity or texture [15]. FCM, K-Means and Otsu segmentation (Figure 2) techniques are considered in this paper.

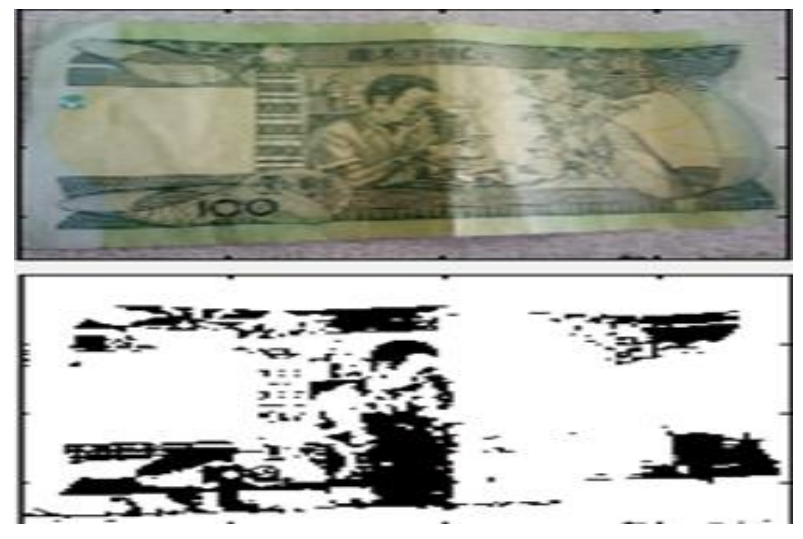

Figure 2. Otsu Segmentation

\section{RESULTS}

The word network in the term 'artificial neural network' refers to the inter-connections between the neurons in the different layers of each system. A system has three layers. The first layer has input neurons, which send data via synapses to the second layer of neurons, and then via more synapses to the third layer of output neurons. More complex systems will have more layers of neurons with some having increased layers of input neurons and output neurons. The synapses store parameters called "weights" that manipulate the data in the calculations [16].

The most popular neural network model is the rnultilayer perceptron (MLP), which is an extension of the single layer perceptron proposed by Rosenblatt. Multilayer perceptrons, in general, are feedforward network, having distinct input, output, and hidden layers.

In this paper, MLP neural network with back propagation is used for classifying Ethiopian paper currencies to their corresponding class. In this experiment, the neural network is tested by SIGMOID and TANH activation functions. The performances of the classifier were tested by ANN (Artificial Neural Network) using three different techniques of segmentation. In order to train the classifiers, $70 \%$ were used for model training and 30\% were used for performance testing. As shown in Figure 3 and 4, the result indicated that there was $91.2 \%$ achieved using BPNN with otsu segmentation. The aim of the research paper is to identify the effects of segmentation techniques in classification of Ethiopian paper currencies. In this paper, BPNN are used and the accuracy of the system is presented, and the results of BPNN with TANH activation function were discussed and hopeful results were obtained. 


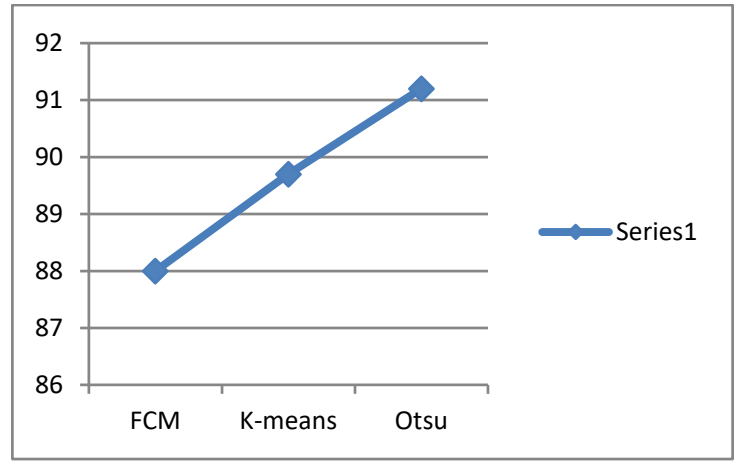

Figure 3. Results of segmentation techniques

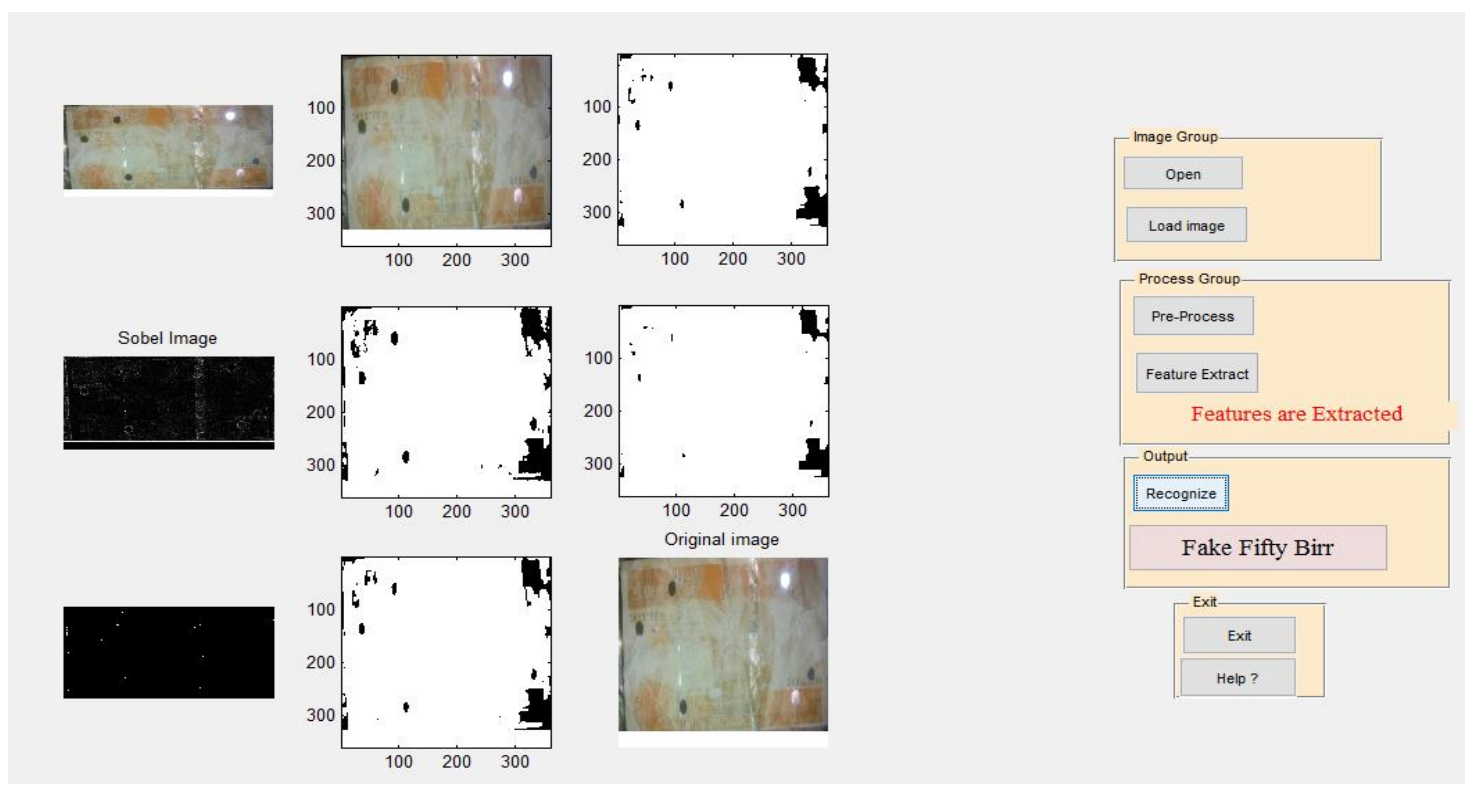

Figure 4. Prototype

\section{ACKNOWLEDGEMENTS}

We greatly acknowledge Bahir Dar University.

\section{REFERENCES}

[1] Jegnaw Fentahun, "Automatic recognition of Ethiopian paper currency", Thesis, Addis Ababa University, 2014.

[2] Zewde Dinku and Kumudha Raimond," Counterfeit Currency Identification System - A Case Study on Ethiopian Birr Note", Addis Ababa University, 2009.

[3] William K. Pratt: Digital image processing, PIKS Scientific inside, John Wiley, 4th Edition, 2007.

[4] Arti Taneja, Priya Ranjan, Amit Ujjlayan “A Performance Study of Image Segmentation Techniques”, IEEE, 2015.

[5] Abrham Debasu Mengistu, Seffi Gebeyehu Mengistu, Dagnachew Melesew Alemayehu, "Image Analysis for Ethiopian Coffee Plant Diseases Identification”, IJBB, 2016.

[6] Ta Yang Goh, Shafriza NishaBasah, HanizaYazid, MuhammadJuhairiAziz Safar, Fathinul SyahirAhmad Saad, "Performance analysis of image thresholding: Otsu technique" ScienceDirect, 2017.

[7] Mahipal Singh Choudhry, Rajiv Kapoor, "Performance Analysis of Fuzzy C-Means Clustering Methods for MRI Image Segmentation”, ScienceDirect, 2016.

[8] NameirakpamDhanachandra, KhumanthemManglem, Yambem JinaChanu, "Image Segmentation Using K -means Clustering Algorithm and Subtractive Clustering Algorithm”, ScienceDirect, 2016.

[9] Yao Yu, Xuesong Suo, "Detection System of Bottle Cap Smoothness Based on Image Processing", TELKOMNIKA, Telecommunication Computing Electronics and Control, 2016.

[10] Jun Sun Jun Sun, Yan Wang Yan Wang, Xiaohong Wu Xiaohong Wu, Xiaodong Zhang, Hongyan Gao, "A New Image Segmentation Algorithm and Its Application in Lettuce Object Segmentation", TELKOMNIKA, Telecommunication Computing Electronics and Control, 2016. 
[11] Lilik Sumaryanti, Aina Musdholifah, Sri Hartati, "Digital Image Based Identification of Rice Variety Using Image Processing and Neural Network", TELKOMNIKA Indonesian Journal of Electrical Engineering, 2015.

[12] Komal Vora, Ami Shah, Jay Mehta, “A Review Paper on Currency Recognition System”, International Journal of Computer Applications, 2015.

[13] Megha Thakur, Amrit Kaur, "Various fake currency detection techniques", International Journal for Technological Research in Engineering, 2014.

[14] Ehsani AR, Shafry MR, Norouzi A. "Digital Dental X-Ray Image Segmentation and Feature Extraction", Indonesian Journal of Electrical Engineering and Computer Science, 2013.

[15] MuhammadSarfraz," An Intelligent Paper Currency Recognition System”, Procedia Computer Science, 2015

[16] Subhadip Basu, Nibaran Das, Ram Sarkar," An MLP based Approach for Recognition of Handwritten 'Bangla' Numerals", International Conference on Artificial Intelligence, 2005. 